\title{
Psychiatric Comorbidities in Patients with Alcohol Dependence Syndrome in a Tertiary Care Center: A Cross-Sectional Study
}

\author{
Bhaskkar Sharma ${ }^{\mathrm{a}, \mathrm{c}}$ Anup Devkota ${ }^{\mathrm{a}, \mathrm{c}}$ Suresh Chandra Pant ${ }^{\mathrm{b}, \mathrm{c}}$
}

\begin{abstract}
:
Introduction: The co-occurence of substance abuse and mental illness is well known. Alcoholics are reported to be three times more likely to suffer from another psychiatric disorder. This study aims to observe the prevalence of psychiatric comorbidity in alcohol dependent patients in our setup. Methods: This was a hospital based crosssectional observational study conducted over a period of three months. Consecutive patients presenting to the outpatient section of Psychiatric department who met the DSM-IV-TR criteria for alcohol dependence were included in the study. They were interviewed using Structured Clinical Interview for DSM-IV-TR (SCID I\&II) to assess for comorbidity. Data was analyzed using Statistical Package for Social Sciences (SPSS TM) software. Mann Whitney U test and Chi square or Fisher's Exact tests were used for statistical analysis. Results: Out of 31 patients enrolled, $14(45.16 \%)$ had psychiatric comorbid conditions all of which were Axis I disorders. Anxiety disorder (35.71\%) was the most frequent associated disorder followed by depressive disorder $(28.57 \%)$. The alcohol dependent patients with comorbidity was significantly younger (mean age $=35.71 \pm 13.60$ years) in comparison to those without comorbidity (mean age $=42.59 \pm 11.15$ years). Other socio-demographic parameters were comparable between the two groups. Patients with history of past psychiatric illness and medication were less likely to have comorbidity, which was statistically significant. Conclusion: Psychiatric comorbidity is common in alcohol dependent people. Anxiety disorder and depression were more prevalent entities. Those with past illness and medications were less likely to have comorbidities.
\end{abstract}

Keywords: Alcohol Dependent, Comorbidity, Psychiatric

\section{INTRODUCTION:}

The co-occurrence of substance abuse and mental illness has been known for long. Alcoholics are reported to be three times more likely to suffer from another psychiatric disorder.[1]

Studies have shown that out of two billion alcohol users worldwide 76.3 million $(7.63 \%)$ have at least one additional disorder caused by their habit. $[2,3,4]$ Alcohol use disorders can also mimic or potentiate most other psychopathological symptoms. [5]

\footnotetext{
Submitted: 16 October, 2018

Accepted: 17 December, 2018

Published: 27 December, 2018

a - Lecturer, Department of Psychiatry

b - Associate Professor and Head, Department of Psychiatry

c - Lumbini Medical College and Teaching Hospital, Pravas, Palpa

Corresponding Author:

Bhaskkar Sharma

e-mail: vasker63@yahoo.com

ORCID: https://orcid.org/0000-0001-8031-6926
}

There has been an increase in the incidence of dual diagnosis in recent years. People with dual diagnosis are more likely to experience disabilities of varying dimensions and severity in comparison to those with a single diagnosis. Chronicity and treatment resistance are reported to be the hallmarks of dual diagnoses.[6]

Studies have shown high association of psychiatric comorbidity with alcohol dependence cases in different settings.[7,8] The superimposition of comorbid psychiatric disorders in those with alcohol dependence adds to the burden on the health care system. In addition, comorbid psychiatric disorders in alcohol dependence are associated with prolonged hospital stay, poor treatment outcomes, higher relapse rates, suicide, homelessness, and

\footnotetext{
$\overline{\text { How to cite this article: }}$

Sharma B. Devkota A. Pant SC. Psychiatric Comorbidities in Patients with Alcohol Dependence Syndrome in a Tertiary Care Center: A Cross-Sectional Study. Journal of Lumbini Medical College. 2018;6(2):5 pages. DOI: 10.22502/jlmc.v6i2.266. Epub: 2018 Dec 27.
} 
negative impact on family.[9]

Alcohol is one of the commonest drugs of abuse in Nepal. Yet, published literature on alcohol dependence and comorbidities is scarce in Nepal. Moreover, findings of the western studies may not be applicable in our culture. This study was therefore carried out to observe the prevalence of psychiatric comorbidity in alcohol dependent patients in our setup.

\section{METHODS:}

This was a hospital based cross-sectional observational study carried out in the department of Psychiatry, Lumbini Medical College and Teaching Hospital (LMCTH). It was conducted over a period of three months from August 2018 to October 2018. Consecutive patients presenting to the out-patient section of department of Psychiatry for the first time and those referred from other departments who met the DSM-IV-TR criteria for alcohol dependence were included in the study.

Ethical approval was obtained from the institutional review committee of LMCTH (IRCLMC) prior to commencement of the study (IRCLMC 02-E/018). Informed written consent was taken from the participants and complete confidentiality was maintained.

The participants were interviewed by consultants using Structured Clinical Interview for DSM-IV-TR (SCID I\&II) to assess for comorbidity. A detailed psychiatric work up was done. Sociodemographic information was recorded in a semistructured preformed proforma. Clinical information noted were history of past psychiatric illness, comorbidity and history of any medications. Detailed history of alcohol habits was taken from the patient and reliability ensured with the attending family member.

Data were entered to Microsoft Excel spread sheet and imported to Statistical Package for Social Sciences (SPSSTM) software version 20 for statistical analysis. Quantitative data were presented in mean \pm SD and qualitative data in frequency and percentages. We used Mann Whitney U test for the analysis of continuous variables and Chi-square test or Fisher's Exact test for categorical variables. P value $<0.05$ was considered statistically significant.

\section{RESULTS:}

A total of 31 patients meeting the criteria for alcohol dependence attended the psychiatric out-patient during the study period. Out of them, nine patients were internal referrals from other departments.

The mean age \pm SD of the study population was $39.48 \pm 12.59$ years. $80.6 \%$ of them were males and $87.1 \%$ were married. $19.35 \%$ patients were unemployed. There were $41.93 \%$ of farmers and $16.12 \%$ migrant laborers working abroad.

Among 31 patients, 14 (45.16\%) patients were found to have comorbid psychiatric illness. The Axis-I comorbidity was found in $45.16 \%$ patients. Axis-II comorbidity (Personality disorders) was not seen in any (Table 1).

Anxiety disorder (35.71\%) was the most frequent Axis I disorder followed by depressive

Table 1. Comorbidity profile in alcohol dependent patients $(n=31)$

\begin{tabular}{lc} 
Diagnosis & Frequency (\%) \\
\hline Axis I only & $14(45.16)$ \\
\hline Axis II only & $0(0)$ \\
Both & $0(0)$ \\
Nil & $17(54.83)$ \\
\hline
\end{tabular}

disorder (28.57\%) associating as a comorbidity in our study population.

The cohort of alcohol dependent patients with comorbidity was significantly younger (mean age $=35.71 \pm 13.60$ years) in comparison to that without comorbidity (mean age $=42.59 \pm 11.15$ years). However this difference was not statistically significant $(p=0.064)$. Similarly, other sociodemographic factors as marital status, employment, education and socioeconomic status did not have any effect on the presence of comorbidity.

Patients with past psychiatric illness showed tendency to have no comorbidity while those who did not have any psychiatric illness were more likely to have comorbid conditions. Similarly those who

Table 2. Common Axis I diagnosis $(n=14)$

\begin{tabular}{lc}
\hline Axis-I Diagnosis & Frequency ( \%) \\
\hline Anxiety disorder & $5(35.71)$ \\
\hline Depressive disorder & $4(28.57)$ \\
\hline Psychotic disorder & $2(14.28)$ \\
\hline Seizure disorder & $2(14.28)$ \\
\hline Conversion disorder & $1(7.1)$ \\
\hline
\end{tabular}


Table 3. Socio-demographic comparison between alcohol dependent patients with and without comorbidity $(n=31)$

\begin{tabular}{|c|c|c|c|c|}
\hline Variables & & $\begin{array}{l}\text { With comorbidity } \\
\qquad(\mathrm{n}=14)\end{array}$ & $\begin{array}{l}\text { Without comorbidity } \\
(\mathbf{n}=17)\end{array}$ & Statistics \\
\hline $\begin{array}{l}\text { Age in years, } \\
\text { Mean } \pm \text { SD }\end{array}$ & & $35.71 \pm 13.60$ & $42.59 \pm 11.15$ & $\begin{array}{c}\text { Mann Whitney } \\
\mathrm{U}=165.5, \mathrm{n}=31 \\
\mathrm{p}=0.064\end{array}$ \\
\hline Gender & $\begin{array}{l}\text { Male } \\
\text { Female }\end{array}$ & $\begin{array}{c}12(38.70 \%) \\
2(6.45 \%)\end{array}$ & $\begin{array}{c}13(41.93 \%) \\
4(12.9 \%)\end{array}$ & $\mathrm{p}=0.664^{*}$ \\
\hline Marital Status & $\begin{array}{l}\text { Single } \\
\text { Married }\end{array}$ & $\begin{array}{c}3(9.7 \%) \\
11(35.48 \%)\end{array}$ & $\begin{array}{c}1(3.22 \%) \\
16(51.61 \%)\end{array}$ & $\mathrm{p}=0.304^{*}$ \\
\hline Employment & $\begin{array}{l}\text { Employed } \\
\text { Unemployed }\end{array}$ & $\begin{array}{c}13(41.93 \%) \\
1(3.22 \%)\end{array}$ & $\begin{array}{l}12(38.71) \\
5(16.12 \%)\end{array}$ & $\mathrm{p}=0.185^{*}$ \\
\hline Education & $\begin{array}{l}\text { Below SLC } \\
\text { SLC and above }\end{array}$ & $\begin{array}{l}9(29.03 \%) \\
5(16.12 \%)\end{array}$ & $\begin{array}{l}10(32.26 \%) \\
7(22.58 \%)\end{array}$ & $\begin{array}{c}\mathrm{X}^{2}=0.097, \mathrm{df}=1, \mathrm{n}=31, \\
\mathrm{p}=0.756\end{array}$ \\
\hline Socioeconomic status & $\begin{array}{l}\text { Low class } \\
\text { Middle class }\end{array}$ & $\begin{array}{l}5(16.12 \%) \\
9(29.03 \%)\end{array}$ & $\begin{array}{l}8(25.80 \%) \\
9(29.03 \%)\end{array}$ & $\begin{array}{c}\mathrm{X}^{2}=0.406, \mathrm{df}=1, \mathrm{n}=31, \\
\mathrm{p}=0.524\end{array}$ \\
\hline
\end{tabular}

* Fisher's Exact Test

had history of past medication were more likely to have no comorbidity (Table 4).

\section{DISCUSSION:}

Table 4. Clinical Comparison between alcohol dependent patients with and without comorbidity $(n=31)$

\begin{tabular}{|c|c|c|c|c|}
\hline Variables & & $\begin{array}{l}\text { With co-morbidity } \\
(n=14)\end{array}$ & $\begin{array}{l}\text { Without co-morbidity } \\
(\mathrm{n}=17)\end{array}$ & Statistics \\
\hline \multirow{2}{*}{$\begin{array}{l}\text { Past psychiatric } \\
\text { illness }\end{array}$} & Yes & $2(6.45 \%)$ & $11(35.48 \%)$ & \multirow{2}{*}{$\begin{array}{l}X^{2}=8.016, d f=1, n=31 \\
p=0.005\end{array}$} \\
\hline & No & $12(38.70 \%)$ & $6(19.35 \%)$ & \\
\hline \multirow{2}{*}{ Past medication } & Yes & $2(6.45 \%)$ & $13(41.93 \%)$ & \multirow{2}{*}{$\begin{array}{l}X^{2}=11.888, d f=1, n=31, \\
p=0.001\end{array}$} \\
\hline & No & $12(38.71 \%)$ & $4(12.9 \%)$ & \\
\hline
\end{tabular}

LMCTH is a tertiary care center catering to the mental health need of a large catchment area encompassing a number of surrounding districts. The present study aimed to observe the prevalence of psychiatric comorbidity in alcohol dependent cases attending the out-patient section of psychiatric department.

The mean age of patients in our study was comparable to another study.[10] Similar to the study by Vohra AK et al.[11] the comorbid group in our study was much younger (mean age $=35.71 \pm 13.60$ years) in comparison to the patients without comorbidity $(42.59 \pm 11.15$ years). However, this difference in mean was not statistically significant $(p=0.064)$. Alcohol dependence syndrome (ADS) and psychiatric comorbidity were more prevalent in males. This is likely because of higher prevalence of ADS, its better awareness and superior status of males.[10] $87.1 \%$ of the enrolled patients were married. As in another study, this might be because married people have better family support and care hence are brought for treatment.[10]

In our study the comorbidity rate was 45.16 $\%$ of which all were Axis I disorders. This prevalence is similar to that of the studies by Chaudhary S. et al.[9] (46.59\%) and Singh A. et al.[12] A French hospital based study by Nubukpo P. et al.[13] also reported a similar prevalence of $42.12 \%$. However, this figure is lower than that of the studies by Vohra AK et al.[11] and Kumar V. et al.[14] in which the prevalence were $76.6 \%$ and $64.8 \%$ respectively. 
Disparity in the sample size and use of diagnostic criteria could explain this variation in the findings. The absence of Axis II disorders in our sample could also be a contributing factor for this lower figure. Moreover, patients in our part of the region tend not to open up regarding their psychiatric or mental issues which leads to a significant chunk of such illness going undetected.

The most frequent Axis I disorder in this study was anxiety disorder (35.71\%) followed by depressive disorder (28.57\%). Similar were the findings in the study done by Shakya DR et al.[10] However, in other studies depressive disorder was more prevalent comorbid condition.[11,14] This contrast could be due to geographical or ethnic variation of the study participants. The findings of this study also implicate that focusing on simultaneous treatment of these comorbid anxiety or mood disorders will result in a better outcome in patients with alcohol dependence.

The co-occurrence of psychotic disorders in our study sample was low (14.28\%) which is in congruence with other studies.[12,15] However some other studies have reported much higher prevalence ranging from $11 \%$ to $25 \%$.[14,16] This is because patients with established or suspected severe psychotic disorders directly present to higher facilities as this center is not a specialized psychiatric hospital.

The present study demonstrates that patients who were diagnosed with some psychiatric illness in the past were less likely to have co-morbidity $(6.45 \%$ vs. $35.48 \%$ ), which was statistically significant. Likewise, those who had undergone medical treatment for psychiatric or non-psychiatric illness were less suffering from co-morbidity $(6.45 \%$ vs. $41.93 \%$ ). This is most likely because these patients tend to be more compliant and motivated, selfconscious regarding their mental health, and more cautious about the presence of psychiatric comorbid conditions.

\section{LIMITATIONS:}

The sample size of our study was small. Only the patients presenting to the out-patient department were included and no controls were taken. Further, this being a facility based study, the generalizability of the study is limited.

\section{CONCLUSION:}

Psychiatric co-morbidity is common in people with alcohol dependence. This study suggested a significant prevalence of psychiatric comorbidity in alcohol dependent patients. Anxiety disorder was the most common comorbidity while no Axis II disorder was observed. Patients with comorbidity were younger compared to their counterparts. Those with past psychiatric illness and medications were less likely to suffer from comorbidity.

\section{Conflict of interest:}

None Declared.

\section{Financial Disclosure:}

No funds were available. 


\section{REFERENCES:}

1. Winokur G. Family history studies: VIII. Secondary depression is alive and well, and... Diseases of the Nervous System. 1972 Feb;33(2):94-9. PMID: 5014298

2. Donadon MF, Osório FL. Personality traits and psychiatric comorbidities in alcohol dependence. Brazilian Journal of Medical and Biological Research. 2016;49(1):e5036. Epub 2015 Nov 27 PMID: 26628399 DOI: 10.1590/1414431X20155036 [Publisher Full Text]

3. Boden JM, Fergusson DM. Alcohol and depression. Addiction. 2011 May;106(5):906-14. PMID: 21382111 DOI: 10.1111/j.1360-0443.2010.03351.x

4. Morley KC, Baillie A, Sannibale C, Teesson M, Haber PS. Integrated care for comorbid alcohol dependence and anxiety and/or depressive disorder: study protocol for an assessor-blind, randomized controlled trial. Addiction Science \& Clinical Practice. 2013 Dec;8(1):19. PMID: 24245491 DOI: $\underline{10.1186 / 1940-0640-8-19}$ [Publisher Full Text]

5. Klimkiewicz A, Klimkiewicz J, Jakubczyk A, Kieres-Salomoński I, Wojnar M. Comorbidity of alcohol dependence with other psychiatric disorders.Part II. Pathogenesis and treatment. Psychiatria Polska. 2015;49(2):277-294. DOI: $\underline{10.12740 / \mathrm{PP} / 26071}$

6. Chukwujekwu CD. Commentary on the Comorbidity of Alcohol Use Disorder and Depression among Patients Attending a Tertiary Hospital in the Niger Delta Region of Nigeria. Journal of Addiction Research and Therapy. 2017;8(319):2. DOI: $\underline{10.4172 / 2155-6105.1000319}$ [Publisher Full Text]

7. Petrakis IL, Gonzalez G, Rosenheck R, Krystal JH. Comorbidity of Alcholism and Psychiatric Disorders: An Overview. Alcohol Research. 2002 Jan 1;26(2):81.

8. Gilder DA, Lau P, Gross A, Ehlers CL. A co-morbidity of alcohol dependence with other psychiatric disorders in young adult Mexican Americans. Journal of Addictive Diseases. 2007 Aug 27;26(4):31-40. PMID: 18032230 DOI: $\underline{10.1300 / J 069 v 26 n 04 \quad 05}$

9. Chaudhury S, Saldanha D, Saini R, Diwan C, Pratap Singh V, Pathak V. Comorbid Psychiatric Disorders in Alcohol Dependence: A Control Study. Journal of Psychiatry.
2018;21(3): 442. DOI: $\underline{10.4172 / 2378-5756.1000442}$ [Publisher Full Text]

10. Shakya DR, Shyangwa P, Sen B. Psychiatric comorbidity in cases admitted for alcohol dependence. Delhi Psychiatry Journal. 2009 Oct;12(2):252-7. [Pubisher Full Text]

11. Vohra AK, Yadav BS, Khurana H. A study of psychiatric comorbidity in alcohol dependence. Indian Journal of Psychiatry. 2003 Oct;45(4):247. PMID: 21206867 [Publisher Full Text]

12. Singh A, Kumar S, Sharma CS, Dixit V, Srivastava RK, Yaduvanshi R. Other psychiatric comorbidities in male patients of alcohol dependence syndrome: a cross sectional study. Indian Journal of Basic and Applied Medical Research. 2016;5(2):828-38. [Publisher Full Text]

13. Nubukpo P, Girard M, Sengelen JM, Bonnefond S, Varnoux A, Marin B et al. A prospective hospital study of alcohol use disorders, comorbid psychiatric) conditions and withdrawal prognosis. Annals of General Psychiatry. 2016 Aug 31;15(1):22. PMID: 27582780 DOI: 10.1186/s12991016-0111-5 [Publisher Full Text]

14. Kumar Vivek, Dalal Pronab K., Trivedi Jitendra K., Kumar Pankaj. A Study of Psychiatric Comorbidity in Alcohol Dependence. Delhi Psychiatry Journal 2010; 13(2): 291-3. [Publisher Full Text]

15. Kattukulathil S, Kallivayalil RA, George R, Kazhungil F. Psychiatric Comorbidity in Alcohol Dependence: A Cross-Sectional Study in a Tertiary Care Setting. Kerala Journal of Psychiatry. 2015 Dec 12;28(2):156-60. [Publisher Full Text]

16. Shantna K, Chaudhury S, Verma AN, Singh AR. Comorbid psychiatric disorders in substance dependence patients: A control study. Industrial Psychiatry Journal. 2009;18(2):84-87. PMID: 21180482 DOI: $10.4103 / 0972-$ 6748.62265. [Publisher Full Text] 